\title{
Switching LPV Controllers for a Variable Speed Pitch Regulated Wind Turbine
}

\author{
Fabien Lescher, Jing-Yun Zhao, Pierre Borne
}

\begin{abstract}
This paper deals with the control of a variable speed, pitch regulated wind turbine in the whole plant operating area. The wind turbine operating area can be divided into several zones, depending on the wind speed, and the control objectives are different for each operating zone. An hybrid control system composed by several LPV controllers which switches during transitions from one operating area to another is designed in order to ensure asymptotic stability and a good level of performances in the whole operating area. The LPV controllers are calculated from a convex LMI formulation of the problem in order to minimize an $H_{2} / H_{\infty}$ criteria that optimizes the energy conversion of the system and that reduces the mechanical fatigue of the plant mechanical structure. The proposed controller is finally compared with two more conventional ones.
\end{abstract}

Keywords: wind energy, LPV modeling, $\mathcal{H}_{2} / \mathcal{H}_{\infty}$ control, switching systems,mechanical fatigue.

\section{Introduction}

Wind energy has widely grown during the last decades and is nowadays the most competitive form of renewable energy. Nevertheless wind energy is not yet cost effective, and consequently, development of new technologies will be crucial for successful penetration of wind energy into electricity market. Implementation of advanced control systems is considered as a promising way to improve wind energy conversion and to decrease wind energy cost. The wind turbine control objectives are mainly to optimize wind energy conversion, and to reduce dynamic loads experienced by the plant mechanical structure. Indeed, dynamic loads hardly affect wind turbine lifetime and mainly determine mechanic components design, and consequently their cost [1].

Designing a control system for a variable speed, pitch regulated wind turbine presents several issues: the system behavior is both highly non linear and quite uncertain, especially because of blades aerodynamic properties which are sensitive to climatic conditions. Moreover, the control purpose is a multiobjective task, because the control system has to optimize a trade off between energy conversion maximization and alleviation of mechanical dynamic loads which result from very lightly damped resonant modes of the structure. The wind turbine operation is also decomposed into several operating zones, depending on the wind speed passing through the rotor: for low wind speeds, the wind energy system has to maximize produced power, whereas for high wind speed, the electric power has to be maintained to the generator nominal power. Another difficulty of this wind turbine control problem is the uncontrollable and stochastic nature of the main component acting on the plant: the wind speed. Moreover, the effective wind speed acting on the whole turbine rotor is a fictitious quantity and is hence not measurable and not available for control operation.

In response to this multivariable and multiobjective control problem, the usual implemented controllers are calculated from a linearization of the model around an operating point by designing several separate and decoupled loops, each loop being tuned for one objective [2][3]. These controllers are therefore non optimal for the multiobjective problem and moreover, this design task has to be repeated for several linearization points on the reference trajectory that the system must follow.

In this paper, a gain-scheduled wind turbine control system is designed for the whole operating region using Linear Matrix Inequalities (LMIs) optimization and the Linear Parameter Varying (LPV) systems 
framework [4]. The system non-linearities inherent to the aerodynamics subsystem are taken into account by an affine quasi-LPV model, obtained from a Jacobian linearization of the system along the reference trajectory. An optimal time-varying controller, calculated through a LMI problem formulation, is calculated in order to minimize an $H_{2} / H_{\infty}$ criterion composed by the different control objectives. This frequency domain criteria optimization is notably known to be efficient to reduce the fatigue of flexible mechanical structure, and is coupled with the output power regulation objective. This controller synthesis procedure has already demonstrated its efficiency for a simpler wind turbine model, especially for mechanical fatigue reduction [5].

Besides, the proposed synthesis methodology also allows to handle the problem of transition between the operating zones of the system, for which the control objectives and the available actuators are not the same. The transitions between the operating zones is a serious problem, which can generate transient phenomena and losses of performance, and moreover this transition occurs frequently during the system operating life. The proposed approach generates an hybrid controller composed by switched LPV controllers which guarantees stability and control performances even during the transition between the controllers, from the construction of a piecewise-affine parameter dependent Lyapunov function [6][7]. The wind turbine system is described in the first part of the paper. The control objectives over the whole operation area are then presented. The LPV modeling of the wind turbine is described, before the hybrid controller synthesis part. The performances of the proposed controller are finally compared with the ones of two other more conventional controllers.

Notations: For two symmetric matrices, $A$ and $B, A>B$ means that $A-B$ is definite positive. $A^{T}$ denotes the transpose of $A$. $\star$ stands for symmetric blocks; $\bullet$ stands for an element that has no influence on the development.

\section{System Description}

The structure of a variable speed, pitch regulated wind energy conversion system is presented in Fig.1. The system is formed by the rotor, the mechanical structure, and by a generator unit, composed by the generator and the static converter connected to the electrical grid. The control system acts on generator in order to apply the reference electromagnetic torque $T_{G, r e f}$ and on the pitch actuator in order to control the pitch angle of the blades $\beta$, calculated from the measurements of the rotational speed of the shaft at the generator side, and of the flexion speed of the tower by an accelerometer located at the tower top.

The effective wind speed $v(t)$ passing through the rotor is considered as a first order dynamic process disturbed by an exogenous signal $m_{v}(t)$

$$
\dot{v}=-\frac{1}{T_{v}} v+m_{v}(t)
$$

with the time constant $T_{v}$ calculated from the stochastic properties of the wind speed [8].

The mechanical model describing the structure of the plant has three degrees of freedom: the flexions of the blades (blades flap motion) and of the tower (tower fore-aft motion) in the direction of the wind, and the torsion of the drive train shaft (Fig.2). A spring-damper representation is used to describe the flexibility of each component. Moreover, the three blades are supposed to move conjointly and to be affected by the same forces at the same time. A linear model of the dynamic behavior of this structure is established using Lagrange's equations.

The electrical subsystem, corresponding to the generation unit, composed by the generator and the power electronic components, has very fast dynamics compared with dynamics of the other subsystems. Consequently, and considering the study objectives, the electrical dynamics are neglected. Hence, electromagnetic torque $T_{G}$ is supposed equal to its reference $T_{G, r e f}$. 


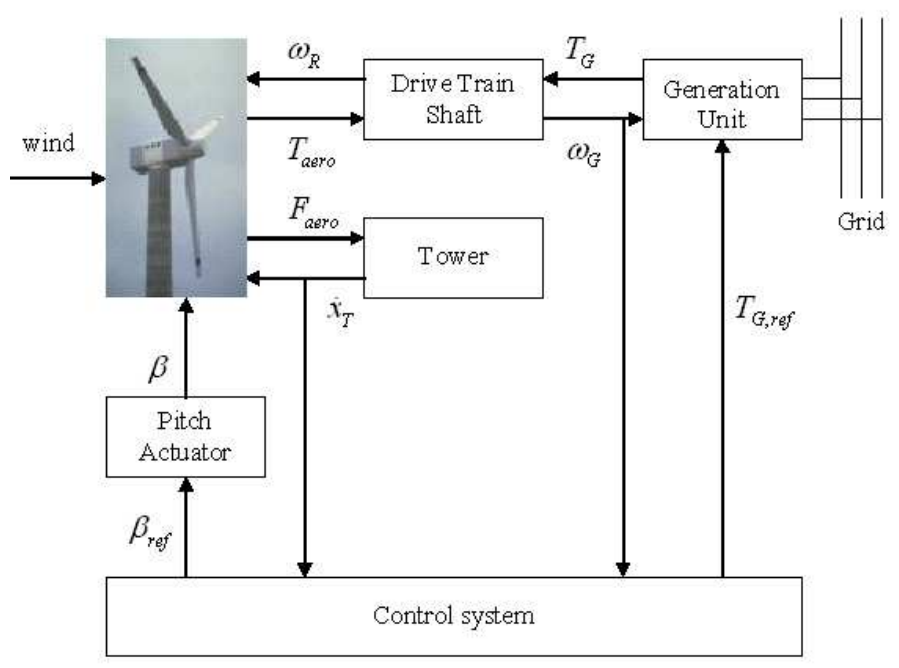

Figure 1: Wind energy conversion system structure.

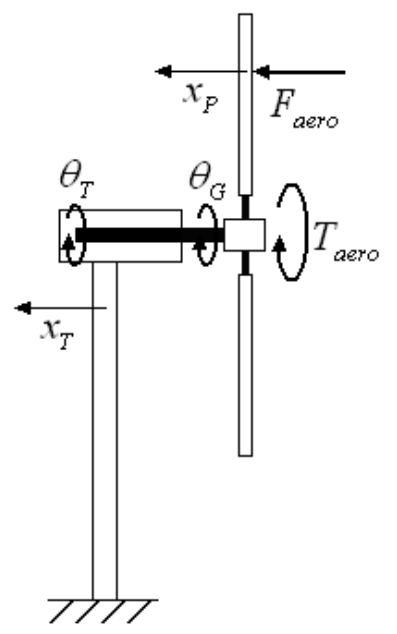

Figure 2: Wind Turbine Mechanical Structure

The pitch actuator subsystem represents the hydraulic or electric system which makes the blades revolve around their lengthwise axis. This system is described by a first order transfer function with a time constant $T_{\beta}$.

The aerodynamic conversion process of the turbine rotor is characterized by the extracted torque $T_{\text {aero }}$ and by the out-of-plane thrust force $F_{\text {aero }}$, which are functions of the air mass density $\rho$, the wind velocity $v$, the rotational speed of the turbine $\omega_{T}$, the horizontal blades speed $\dot{x}_{P}$ and the power and thrust coefficients $C_{p}$ and $C_{t}$ :

$$
\begin{gathered}
T_{\text {aero }}=\frac{1}{2} \rho \pi R^{2} \frac{\left(v+\dot{x}_{P}\right)^{3}}{\omega_{T}} C_{p}(\lambda, \beta), \\
F_{\text {aero }}=\frac{1}{2} \rho \pi R^{2}\left(v+\dot{x}_{P}\right)^{2} C_{t}(\lambda, \beta),
\end{gathered}
$$

with $R$ the length of the rotor blades. The aerodynamic coefficients $C_{p}$ and $C_{t}$ are non-linear functions depending on blades pitch angle $\beta$ and tip speed ratio $\lambda=\frac{\omega_{T} R}{v}$. 


\section{Control Task}

The controller objectives are to ensure:

- stability along the reference trajectory for the whole operation,

- good performances of the selected outputs, i.e. energy conversion and alleviation of mechanical loads affecting the plant structure.

The wind turbine operation area can be divided into three zones, depending on the wind speed acting on blades. The energy conversion objectives, and thus the control objectives, are different for each zone.

For low wind speed, i.e. for $v<v_{1}$, the main objective is to maximize system energy conversion yield. In this Partial Load 1 zone, system has to operate at $C_{p}(\lambda, \beta)=C_{p, \max }$. Pitch angle $\beta$ is then maintained constant at $\beta_{o p t}$ and rotational speed $\omega_{T}$ is controlled to minimize the criteria $\delta \lambda=\lambda-\lambda_{\text {opt }}$, by acting only on generator electromagnetic torque $T_{G}$.

For higher wind speed, corresponding to $v_{1}<v<v_{2}$, turbine rotational speed $\omega_{T}$ is maintained at the nominal generator speed by acting on electromagnetic torque $T_{G}$. Pitch angle $\beta$ is also maintained at $\beta_{\text {opt }}$ to maximize energy conversion efficiency (Partial Load 2).

For high wind speed, i.e. $v>v_{2}$, wind turbine operates in Full Load and electric produced power $P_{\text {elec }}$ has to be regulated at nominal generator power. Turbine rotational speed is maintained around nominal generator speed and pitch angle $\beta$ is controlled in order to reduce power coefficient $C_{p}(\lambda, \beta)$. Control system is then multivariable in this zone, because it acts on both generator torque and pitch angle.

Evolution of the main variables in function of wind speed are presented in Fig.3. The control system has

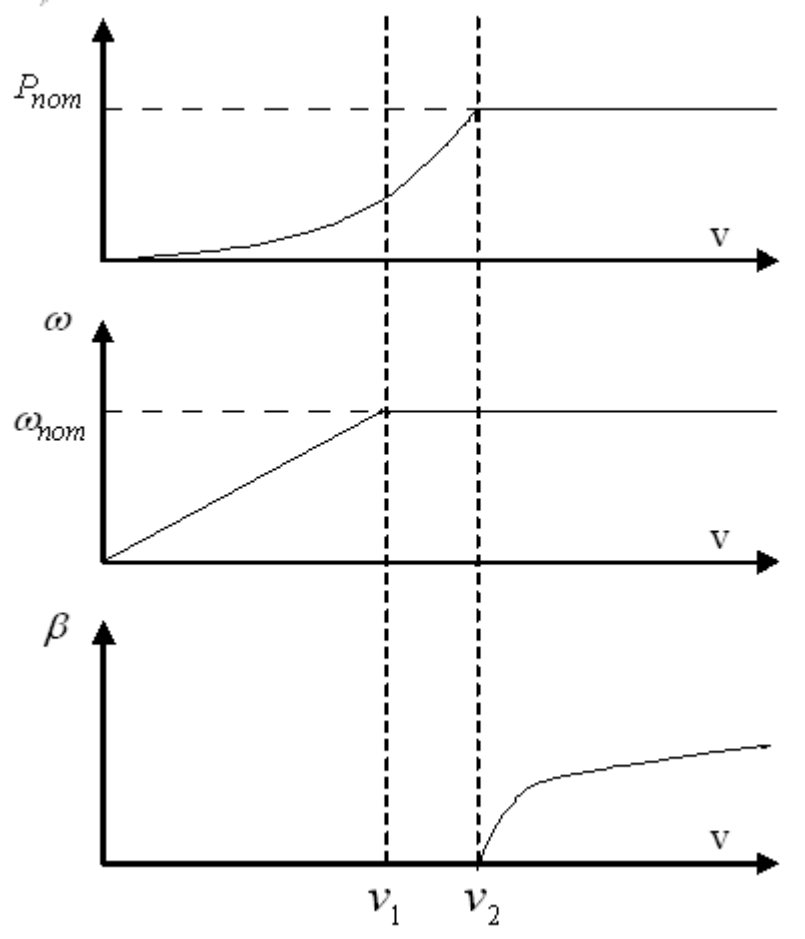

Figure 3: Evolution of the main variables in function of wind speed.

to operate over the full envelope of wind speeds. Hence, transitions between these different operating zones have to be handled by the control system in a smooth manner, which avoids the generation of large transients. 
The other main control objective is to reduce mechanical fatigue of most expensive plant components, especially blades, drive train and tower. Hence, the control system will be designed in order to reduce the variations of the drive train torsion torque $T_{D}$ and the blades and tower flexion forces $F_{B}$ and $F_{T}$, expressed by:

$$
\begin{aligned}
& T_{D}=d_{D}\left(\omega_{T}-\omega_{G}\right)+k_{D}\left(\theta_{T}-\theta_{G}\right) \\
& F_{B}=d_{B} \dot{x_{B}}+k_{B} x_{B} \\
& F_{T}=d_{T} \dot{x_{T}}+k_{B} x_{T}
\end{aligned}
$$

with $d$ and $k$ the damper and spring coefficients of the corresponding components.

The control system will be designed in order to optimize a trade-off between these objectives by minimizing a criterion expressed in the frequency range. $H_{2}$ norm optimization permits to reduce the average variations of the energy conversion parameter, i.e. $\delta \lambda, \delta \omega_{T}$ or $\delta P_{\text {elec }}$, in the whole frequency range. $H_{\infty}$ cost minimization is able to alleviate, in the frequency range, the maximum response of the mechanical loads to a variation of wind speed, which is generally the response corresponding to the resonance frequency of the component, and which is the most damaging.

\section{LPV Modeling}

The LPV model can be considered as a group of linear local descriptions of nonlinear descriptions. Given the nonlinear system describing the wind turbine behavior

$$
\begin{aligned}
\dot{x} & =f(x, u, w) \\
y & =g(x, u, w) \\
z_{2} & =h_{2}(x, u, w) \\
z_{\infty} & =h_{\infty}(x, u, w)
\end{aligned}
$$

with $x$ the state of the system, $u$ the control input, $w$ the external disturbance, $y$ the measured output and $z_{2}$ and $z_{\infty}$ the performance outputs, the Jacobian linearization approach can be used to create an LPV system based on the first-order Taylor series expansion of the nonlinear model. A family of linear plants is obtained by linearizing the nonlinear system with respect to a set of equilibrium points located on the reference trajectory, which are parametrized by the scheduling parameter $\rho$ and satisfy $f\left(x_{e}(\rho), u_{e}(\rho), 0\right)=0$. Corresponding to a specified family of equilibrium points, the family of the linearized plants can be written in the following form:

$$
\left[\begin{array}{c}
\delta \dot{x} \\
\delta y \\
\delta z_{2} \\
\delta z_{\infty}
\end{array}\right]=\left[\begin{array}{ccc}
\left.\frac{\partial f}{\partial x}\right|_{e} & \left.\frac{\partial f}{\partial u}\right|_{e} & \left.\frac{\partial f}{\partial w}\right|_{e} \\
\left.\frac{\partial g}{\partial x}\right|_{e} & \left.\frac{\partial g}{\partial u}\right|_{e} & \left.\frac{\partial g}{\partial w}\right|_{e} \\
\left.\frac{\partial h_{2}}{\partial x}\right|_{e} & \left.\frac{\partial h_{2}}{\partial u}\right|_{e} & \left.\frac{\partial h_{2}}{\partial w}\right|_{e} \\
\left.\frac{\partial h_{\infty}}{\partial x}\right|_{e} & \left.\frac{\partial h_{\infty}}{\partial u}\right|_{e} & \left.\frac{\partial h_{\infty}}{\partial w}\right|_{e}
\end{array}\right]\left[\begin{array}{c}
\delta x \\
\delta u \\
\delta w
\end{array}\right]
$$

where the deviation variables are defined by

$$
\begin{aligned}
\delta x & =x-x_{e}(\rho) \\
\delta u & =u-u_{e}(\rho) \\
\delta y & =y-y_{e}(\rho) \\
\delta w & =w-w_{e}(\rho)
\end{aligned}
$$

and where $\left.J\right|_{e}$ represents the value of the Jacobian coefficient $J$ at the equilibrium point $\left(x_{e}(\rho), u(\rho)\right)$. Before deriving the LPV model of the wind turbine, the scheduling parameters must be selected so 
that the appropriate equilibrium point can be located on the reference trajectory. Because the reference trajectory is decomposed into three parts, corresponding to the three operating areas, the parameter set $\mathcal{P}=\left[\rho_{\min }, \rho_{\max }\right]$ is partitioned into three subsets

$$
\begin{aligned}
& \mathcal{P}_{1}=\left[\rho_{1, \text { min }}, \rho_{1, \text { max }}\right] \\
& \mathcal{P}_{2}=\left[\rho_{2, \text { min }}, \rho_{2, \text { max }}\right] \\
& \mathcal{P}_{3}=\left[\rho_{3, \text { min }}, \rho_{3, \text { max }}\right]
\end{aligned}
$$

with $\rho_{1, \max }=\rho_{2, \min }$ and $\rho_{2, \max }=\rho_{3, \min }$ corresponding to $v_{1}$ and $v_{2}$, i.e. the transitions between the different operating regions.

Hence, by calculating the Jacobian coefficients as functions of the scheduling parameter $\rho$ along the reference trajectory for each parameter subset $\mathcal{P}_{i}$, and considering the wind turbine system configuration, the dynamic behavior of the model (4) for the wind turbine system is governed by the equation:

$$
\left[\begin{array}{c}
\delta \dot{x} \\
\delta y \\
\delta z_{2} \\
\delta z_{\infty}
\end{array}\right]=\left[\begin{array}{ccc}
A_{i}(\rho) & B_{i} & G_{i} \\
C_{y, i}(\rho) & 0 & 0 \\
C_{z_{2}, i}(\rho) & D_{z_{2}, i}(\rho) & 0 \\
C_{z_{\infty}, i}(\rho) & D_{z_{\infty}, i}(\rho) & 0
\end{array}\right]\left[\begin{array}{c}
\delta x \\
\delta u \\
\delta w
\end{array}\right]
$$

$i=\{1,2,3\}$.

The Jacobian coefficients of the expression of the aerodynamic torque and of the thrust force (2) are calculated along the reference trajectory and are approximated by affine functions of the scheduling parameter $\rho$. Hence matrices $A_{i}(\rho)$ have the following form:

$$
A_{i}(\rho)=A_{i, 0}+\rho A_{i, 1}
$$

and are continuous in the whole parameter set $\mathcal{P}$.

\section{LPV Control Design}

From the developed family of LPV models of the wind turbine, we aim at designing a family of LPV dynamic output-feedback controllers in the form:

$$
\left[\begin{array}{c}
\dot{x}_{K} \\
u
\end{array}\right]=\left[\begin{array}{cc}
A_{K, i}(\rho) & B_{K, i}(\rho) \\
C_{K, i}(\rho) & D_{K, i}(\rho)
\end{array}\right]\left[\begin{array}{c}
x_{K} \\
y
\end{array}\right], i=\{1,2,3\}
$$

The order of each controller is the same than the plant order, and the controllers dynamics are allowed to be discontinuous at the boundaries of the subsets $\mathcal{P}_{i}$.

Hence one LPV controller is designed in each subset $\mathcal{P}_{i}$ in order to optimize a set of performances, expressed as a multichannel $H_{2} / H_{\infty}$ criteria, corresponding to the control objectives of the operating area. The control synthesis is based on the LMI optimization and on the construction of a continuous piecewise-affine Lyapunov function depending on the scheduling parameter $\rho$, which mimics the parameter dependence of the plant model (6). Hence, the parameter dependent Lyapunov function is defined as:

$$
V\left(x_{c l}, \rho\right)=x_{c l}^{T} P(\rho) x_{c l}
$$

where $x_{c l}^{T}=\left(\begin{array}{ll}x^{T} & x_{K}^{T}\end{array}\right)$ and $P(\rho)=\phi_{i}(\rho) P_{i}(\rho)$, with $\phi_{i}(\rho)=1$ if $\rho \in \mathcal{P}_{i}$, and $\phi_{i}(\rho)=0$ otherwise. In order to apply the linearizing changing of variables described in [9], matrices $P_{i}(\rho)$ and $P_{i}^{-1}(\rho)$ are partitioned as:

$$
P_{i}(\rho)=\left(\begin{array}{cc}
Y_{i}(\rho) & S_{i}(\rho) \\
S_{i}^{T}(\rho) & \bullet
\end{array}\right) \quad P_{i}^{-1}(\rho)=\left(\begin{array}{cc}
X & R \\
R^{T} & \bullet
\end{array}\right)
$$


with $I-Y_{i}(\rho) X=S_{i}(\rho) R^{T}$. As explained in [4], matrices $X$ and $R$ are constrained to be constant over the whole parameter set $\mathcal{P}$ in order to obtain a family of controllers independent of the gain-scheduling parameter rate $\dot{\rho}$, which is not available in real-time. Moreover, matrices $Y_{i}(\rho)$ are affine in the parameter $\rho$ in each subset $\mathcal{P}_{i}$ :

$$
Y_{i}(\rho)=Y_{i, 0}+\left(\rho-\rho_{i, \min }\right) Y_{i, 1}
$$

and the function $Y(\rho)=\phi_{i}(\rho) Y_{i}(\rho)$ is constrained to be continuous over the whole parameter set $\mathcal{P}$ :

$$
Y_{i}(\rho)=Y_{j}(\rho) \forall \rho \in \mathcal{P}_{i} \bigcap \mathcal{P}_{j}
$$

By verifying that for the wind turbine model the triple $\left(A_{i}(\rho), B_{i}(\rho), C_{y, i}(\rho)\right)$ is stabilizable and detectable for all $\rho \in \mathcal{P}$, and by assuming that the scheduling parameter rate $\dot{\rho}$ is bounded in a set $\left[-\dot{\rho}_{\max }, \dot{\rho}_{\max }\right]$, the family of controllers can be calculated by applying the following theorem ${ }^{1}$ :

Theorem:[9][6] Suppose there exist symmetric definite positive matrices $X$ and $Y$ such as (10) and (11), matrices

$$
\begin{aligned}
\hat{A}_{i}(\rho) & =\hat{A}_{i, 0}+\left(\rho-\rho_{i, \text { min }}\right) \hat{A}_{i, 1}+\left(\rho-\rho_{i, \min }\right)^{2} \hat{A}_{i, 2} \\
\hat{B}_{i}(\rho) & =\hat{B}_{i, 0}+\left(\rho-\rho_{i, \text { min }}\right) \hat{B}_{i, 1}+\left(\rho-\rho_{i, \text { min }}\right)^{2} \hat{B}_{i, 2} \\
\hat{C}_{i}(\rho) & =\hat{C}_{i, 0}+\left(\rho-\rho_{i, \text { min }}\right) \hat{C}_{i, 1} \\
\hat{D}_{i}(\rho) & =\hat{D}_{i, 0}+\left(\rho-\rho_{i, \text { min }}\right) \hat{D}_{i, 1}
\end{aligned}
$$

symmetric semi-definite positive matrices

$$
M_{i}=\left(\begin{array}{cc}
M_{1, i} & \star \\
M_{2, i} & M_{3, i}
\end{array}\right)
$$

and a symmetric matrix $Q$ satisfying the following matrix inequalities

$$
\begin{aligned}
& \left(\begin{array}{cc}
U+U^{T} & \star \\
\hat{A}_{i}+\left(A_{i}+B_{i} \hat{D}_{i} C_{y, i}\right)^{T} & \dot{\rho} Y_{i, 1}+V+V^{T} \\
G^{T} & G^{T} Y_{i} \\
C_{z_{\infty}} X+D_{z_{\infty}} \hat{C}_{i} & C_{z_{\infty}}+D_{z_{\infty}} \hat{D}_{i} C_{y, i}
\end{array}\right. \\
& \left.\begin{array}{cc}
\star & \star \\
\star & \star \\
-\gamma I & \star \\
0 & -\gamma I
\end{array}\right)+\left(\rho-\rho_{i, \min }\right)^{2}\left(\begin{array}{cc}
M_{i} & 0 \\
0 & 0
\end{array}\right)<0 \\
& \left(\begin{array}{ccc}
X & \star & \star \\
I & Y_{i} & \star \\
D_{z_{2}} \hat{C}_{i}+C_{z_{2}} X & C_{z_{2}}+D_{z_{2}} \hat{D}_{i} C_{y, i} & Q
\end{array}\right)>0 \\
& \operatorname{Tr}(Q)<v \\
& \left(\begin{array}{cc}
M_{1, i} & \star \\
M_{2, i}+\hat{A}_{i, 2} & M_{3, i}+Y_{i} A_{i}+\hat{B}_{i, 2} C_{y, i}
\end{array}\right)>0
\end{aligned}
$$

for all pairs $(\rho, \dot{\rho}) \in\left\{\rho_{i, \min }, \rho_{i, \max }\right\} \times\left\{\dot{\rho}_{i, \min }, \dot{\rho}_{i, \max }\right\}$, with

$$
\begin{aligned}
U & =A_{i} X+B_{i} \hat{C}_{i} \\
V & =Y_{i} A_{i}+\hat{B}_{i} C_{y, i}
\end{aligned}
$$

\footnotetext{
${ }^{1}$ the dependance on $\rho$ is dropped for the notation conveniance
} 
Then the family of dynamic controllers (7) with, $\forall \rho \in \mathcal{P}$ :

$$
\begin{aligned}
D_{K, i}= & \hat{D}_{i} \\
C_{K, i}= & \left(\hat{C}_{i}-D_{K, i} C_{y, i} X\right) R^{-T} \\
B_{K, i}= & S_{i}^{-1}\left(\hat{B}_{i}-Y_{i} B_{i} D_{K, i}\right) \\
A_{K, i}= & S_{i}^{-1}\left(\hat{A}_{i}-S_{i} B_{K, i} C_{y, i} X-Y_{i} B_{i} C_{K, i} R^{T}\right. \\
& \left.-Y\left(A_{i}+B_{i} D_{K, i} C_{y, i}\right) X\right) R^{-T}
\end{aligned}
$$

where matrices $R$ and $S_{i}$ verify for all $\rho \in \mathcal{P}$ the relation $R S_{i}^{T}=I-X Y_{i}$, guarantees that:

- the elements of the family of closed-loop systems composed by (5) and (7) are asymptotically stable;

- the $H_{\infty}$-norm of the transfer $w \rightarrow z_{\infty}$ is less than $\sqrt{\gamma}$;

- the $H_{2}$-norm of the transfer $w \rightarrow z_{2}$ is less than $\sqrt{v}$.

Note that the LMI problem formulated in this theorem has a finite number of inequalities, thanks to the introduction of matrices $M_{i}$, and permits to do without the gridding phase which is commonly used for LPV synthesis and which is very computationally intensive.

Moreover, in order to facilitate the control system implementation, and to prevent the system from having too fast dynamics, additional LMIs constraints are formulated to place the poles of the closed loop systems in a determined region of the state-space domain. Placing the poles in a circle centered at the origin of the state-space permits to prevent for obtaining too fast poles and too few damped fast poles. Hence if the following LMIs are satisfied for $\forall \rho \in\left\{\rho_{i, \min }, \rho_{i, \max }\right\}$ and for semi-definite positive matrices $N_{i}=\left(\begin{array}{cc}N_{1, i} & \star \\ N_{2, i} & N_{3, i}\end{array}\right):$

$$
\begin{aligned}
& \left(\begin{array}{cccc}
-r X & \star & \star & \star \\
-r I & -r Y_{i} & \star & \star \\
A_{i} X+B_{i} \hat{C}_{i} & \hat{A}_{i} & -r X & \star \\
A_{i}+B_{i} \hat{D}_{i} C_{y, i} & Y_{i} A_{i}+\hat{B}_{i} C_{y, i} & -r I & -r Y_{i}
\end{array}\right) \\
& +\left(\rho-\rho_{i, \min }\right)^{2}\left(\begin{array}{cc}
N_{i} & 0 \\
0 & N_{i}
\end{array}\right)<0 \\
& \left(\begin{array}{cccc}
N_{1, i} & \star & \star & \star \\
N_{2, i} & N_{3, i} & \star & \star \\
0 & \hat{A}_{i, 2} & N_{1, i} & \star \\
0 & \hat{B}_{i, 2} C_{y, i}+Y_{i, 1} A_{i, 1} & N_{2, i} & N_{3, i}
\end{array}\right)>0
\end{aligned}
$$

then the poles of the closed-loop systems composed by (5) and (7) are located in a circle of rayon $r$ and of center the origin of the state-space.

Hence, an optimal family of dynamic controllers that minimize the $H_{2}$-norm of the transfer $w \rightarrow z_{2}$ for a given $H_{\infty}$-norm $\sqrt{(\gamma)}$ of the transfer $w \rightarrow z_{\infty}$ can be designed by solving the following convex LMIs problem:

$$
\min v \text { subject to the LMIs (12) and (13) }
$$

\section{Wind Turbine Control System Design and Simulation}

The wind turbine control system is designed from the family of developed LPV systems by calculating LPV controllers which optimize a trade-off between the energy conversion, i.e. the energy yield 
maximization in Partial Load and the output energy regulation in Full Load, and the reduction of mechanical loads. Hence the performance output $z_{2}$ represents the energy optimization variables $\delta \lambda, \delta \omega_{T}$ or $\delta P_{\text {elec }}$, depending on the operating area, and then the subset of the scheduling parameter, augmented by the corresponding weighting functions. In the Full Load operation, and in order to reduce the pitch actuator fatigue, the pitch angle deviation $\delta \beta$ is added to this vector. The performance output vector $z_{\infty}$ is composed by the mechanical forces affecting the plant structure, $F_{T}, F_{P}$ and $T_{D}$, augmented by the corresponding weighting functions. In order to reduce the vibrations of the mechanical components for frequencies above their resonance frequencies, the outputs $F_{T}, F_{P}$ and $T_{D}$ are multiplied by a high-pass filter, in order to penalize the high-frequency variations of these outputs during the controller synthesis. Inversely, the energy optimization variables are multiplied by a low-pass filter in order to ensure to the energy conversion output a good tracking at low frequency. Indeed, at high frequency, a good tracking of these components is not reached because it would induce an increase of dynamical loads on the actuators and on the mechanical structure.

The scheduling parameter $\rho$ is chosen to be $\rho=P_{\text {elec }}+k \beta^{2}, k>0$, because it permits to locate the operating point on the reference trajectory and is directly measured, contrary to the wind speed crossing over the turbine rotor. Moreover, the bounds of the scheduling parameter rate $\dot{\rho}$ are derived from the actuators rate limitations.

By solving numerically the problem (14), three LPV controllers are designed, one for each operating area. Therefore, the control applied to the plant actuators can be discontinuous during controllers switching. Because the switchings between Partial Load 2 and Full Load occur for $\beta=0$, the pitch control is ensured to be continuous, whereas no continuity guarantees are provided concerning the generator torque. To ensure this, a structural constraint on the controllers matrices is added during the synthesis of the LPV controllers: the lines of the controllers matrices $C_{K, i}$ and $D_{K, i}$ corresponding to the generator torque are constrained to be equal at the boundaries of the subsets $\mathcal{P}_{i}$ :

$$
\begin{aligned}
C_{K, i(1)}(\rho) & =C_{K, j(1)}(\rho) \\
D_{K, i(1)}(\rho) & =D_{K, j(1)}(\rho)
\end{aligned}
$$

for all $\rho \in \mathcal{P}_{i} \cap \mathcal{P}_{j}$.

The efficiency of the proposed controller is compared, at the sight of simulation results, with the ones of the two other existing controllers, a gain scheduling PI-based controller, and a multivariable gain scheduling LQG one, for the Full Load operation. Indeed, this operating area is the most challenging in the viewpoint of control design because the controller is multivariable, because the mechanical loads affecting the plant are the highest, and because the pitch action is really efficient to alleviate these loads with a carefully designed controller [2].

As mentioned in [2] and [3], the PI-based controller is designed by calculating controllers for two separate loops: firstly, a PI controller is tuned to guarantee power regulation from generator speed measurement. Then a tower speed feedback is designed to increase tower fore-aft damping, by calculating a controller which appropriately filters blades flap excitation and which does not interact with the bandwidth of the first loop. Unlike the proposed controller, this controller acts only on pitch angle.

The gain scheduling LQG controller is designed from the same augmented model and with the same performances outputs as the proposed controller. The LQG methodology permits to design a multivariable controller which optimizes a time domain quadratic criteria representing a trade off between the different control objectives. Unlike the proposed LPV design methodology, the LQG design does not provide guarantees of stability and performances along the reference trajectory, but only in several operating points on this trajectory. Actually, one LQG controller has to be designed at each linearization point of the trajectory, and a gain scheduling process has to be used to interpolate the different LQG controllers. Moreover, and contrary to the proposed method, the LQG design does not provide any specification of performance in the frequency range such as $H_{\infty}$ or $H_{2}$ norms. 

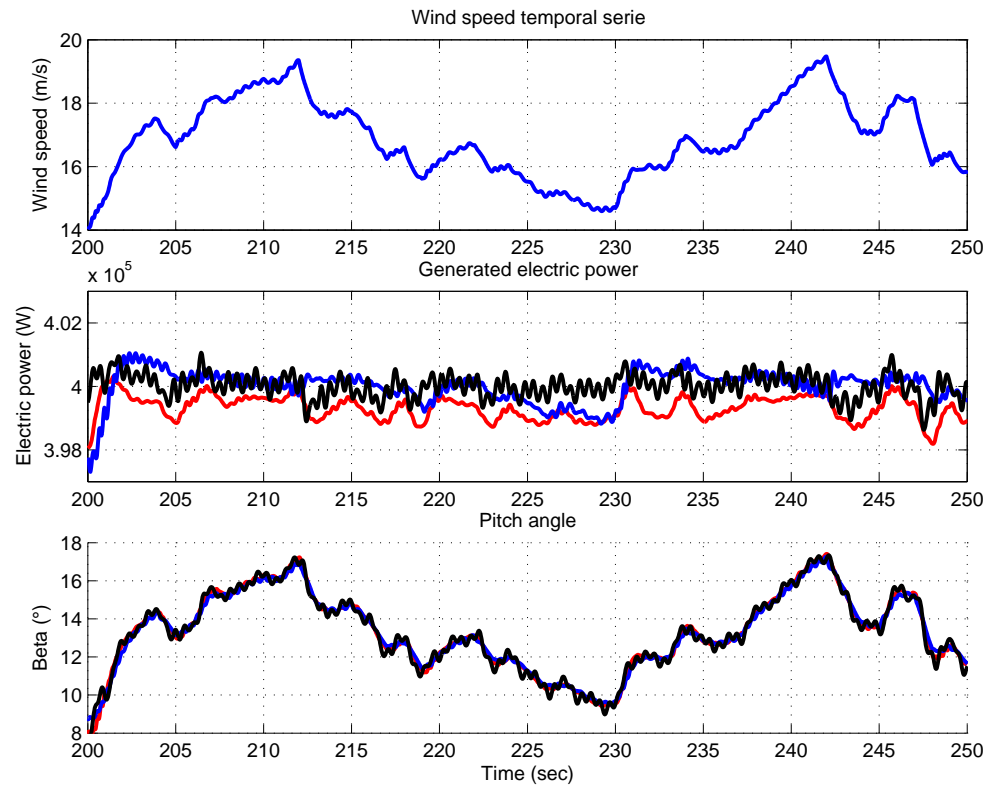

Figure 4: Temporal series. - LPV controller; - PI-controller; - LQG controller.

Table 1: Equivalent Load: Ratio between the different controllers and PI controller.

\begin{tabular}{|l||c|c|c|}
\hline Controller & Shaft & Tower & Blades \\
\hline LPV controller & $57.3 \%$ & $63.8 \%$ & $82.9 \%$ \\
\hline LQG controller & $59.0 \%$ & $73.4 \%$ & $87.8 \%$ \\
\hline PI controller & $100 \%$ & $100 \%$ & $100 \%$ \\
\hline
\end{tabular}

The simulations are based on a dynamic model implemented in Matlab-Simulink of a three blades 400 $\mathrm{kW}$ wind turbine containing:

- non linear and stationary aerodynamics, with rotational effects disturbances due to wind shear and tower shadow.

- flexible drive train model, flexible tower model including first fore-aft mode, flexible blades in flapwise direction,

- pitch actuator limitations on pitch rate $\left( \pm 10^{\circ} / \mathrm{s}\right)$ and pitch amplitude $\left(0^{\circ}-30^{\circ}\right)$.

The simulated wind speed respects stochastic properties of Van der Hoven spectra with high fluctuations. The controllers performances are compared for both power regulation and alleviation of mechanical fatigue. These controllers have equivalent bandwiths and are tuned in order to guarantee a similar level of performance for power regulation. Evaluation of mechanical fatigue is provided by using the Rainflow Counting Algorithm, which calculates the number of load cycles from time domain simulations results, and the fatigue equivalent load for each component [10].

Temporal series of wind speed, produced power $P_{\text {elec }}$ and pitch angle $\beta$ are presented in Fig.4. Fatigue equivalent loads for the shaft, blades and tower are calculated from the simulations, and Table 1 presents the ratio between the equivalent loads obtained with the different controllers and with the PI-controller.

The proposed LPV controller is seen to be more effective for the two selected control objectives, especially for mechanical fatigue reduction for each component, and despite a pitch activity inferior to the pitch activity caused by the LQG controller. A sensitive gain of lifetime of the mechanical components of the plant, or a sensitive reduction of the mass, and consequently of the cost of these components can 

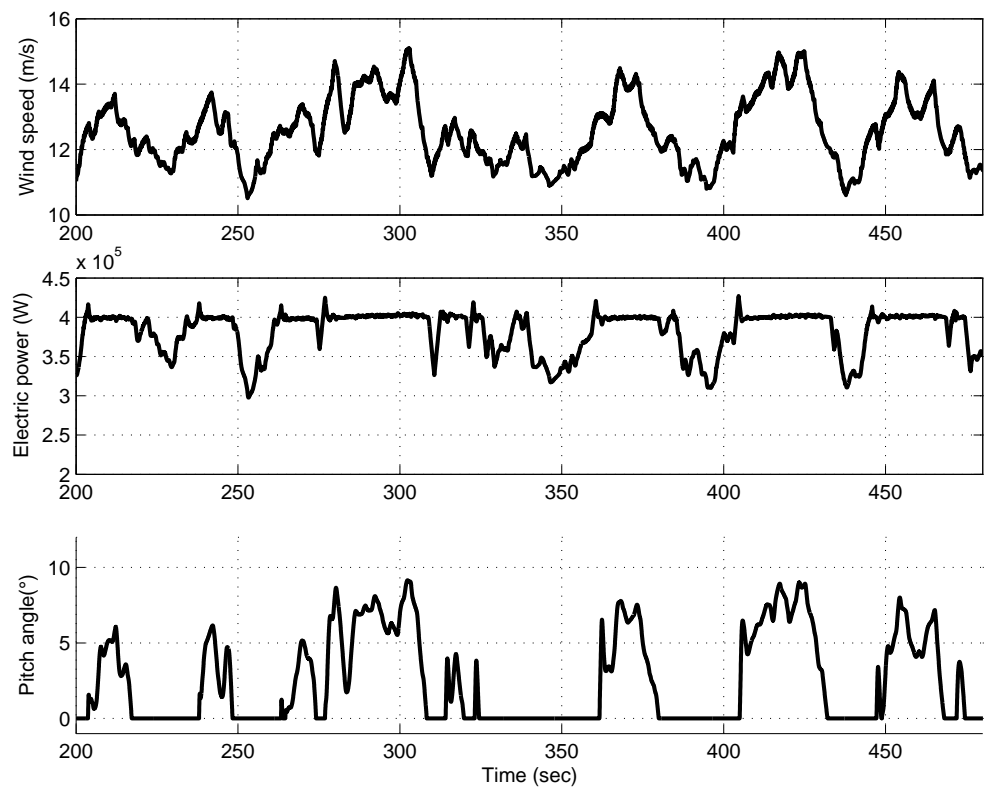

Figure 5: Transitions between Partial Load 2 and Full Load.

then be expected.

The behavior of the plant with the proposed control system is also shown during the transition between Partial Load 2 and Full Load in Fig.5, i.e. for wind speeds around the rated wind speed, which is about $12.2 \mathrm{~m} / \mathrm{s}$. The control system is then seen to handle efficiently this transition.

\section{Conclusion}

In this paper, an hybrid controller composed by several LPV controllers has been proposed for the whole operating area of a variable speed, pitch regulated wind turbine. This control system achieves the optimization of a trade-off between the energy conversion and the reduction of the mechanical loads for the whole envelope of wind speeds acting on the plant, taking into account the different objectives of the energy conversion for the different operating areas.

Moreover, the employed LPV modeling, as well as the LMI formulation of the problem, provides a good framework for additional possible constraints of the control problem during the synthesis, like actuator saturation, or for ensuring the robustness to the system parameters uncertainties.

\section{References}

[1] T. Burton, D. Sharpe, N. Jenkins, and E. Bossanyi, Wind Energy Handbook. John Wiley and Sons, 2001.

[2] E.A. Bossanyi, The design of Closed Loop Controllers for Wind Turbines, Wind Energy, vol.3, 2001, pp.149-163.

[3] W.E. Leithead, S. Dominguez and C.J. Spruce, Analysis of Tower/Blade interaction in the cancellation of the tower fore-aft mode via control, Proc. European Wind Energy Conference, London, UK, 2004. 
[4] P. Apkarian and R. J. Adams, ŞAdvanced gain-scheduling techniques for uncertain systems, Transactions on Control System Technology, vol. 6, pp. 21-32, 1997.

[5] F. Lescher, J. Zhao, and P. Borne, ŞRobust gain scheduling controller for pitch regulated variable speed wind turbine, Ť Studies in Informatics and Control, vol. 14, pp. 299-315, 2005.

[6] S. Lim, Analysis and Control of Linear Parameter-Varying Systems, Ph.D. Dissertation, Stanford University, 1999.

[7] B. Lu and F. Wu, Switching LPV control designs using multiple parameter-dependent Lyapunov functions, Automatica, vol.40, 2004, pp. 1973-1980.

[8] C. Nichita, D. Luca, B. Dakyo, and E. Ceanga, ŞLarge band simulation of the wind speed for real time wind turbine simulators, $\breve{T}$ IEEE Transactions on Energy Conversion, vol. 17, pp. 523-529, 2002.

[9] C. Scherer, P. Gahinet, and M. Chilali, ŞMultiobjective outputfeedback control via lmi optimization, Ť IEEE Transactions on Automatic Control, vol. 42, pp. 896-910, 1997.

[10] M. Matsuiski and T.Endo, Fatigue of metals subjected to varying stress, Japan Soc. Mech. Engrg, 1969.

F. Lescher and P. Borne

Ecole Centrale de Lille

LAGIS Laboratory

BP 48, Cité Scientifique, 59651 Villeneuve d'Ascq Cedex, France

E-mail: fabien.lescher@eigsi.fr

J.Y. Zhao

EIGSI La Rochelle

ERPA Laboratory

26 rue Vaux Le Foletier, 17000 La Rochelle, France

E-mail: jing-yun.zhao@eigsi.fr

Received: November 2, 2006 